\title{
Si PIN X-ray photon counter
}

\author{
$\operatorname{AUTHOR}(\mathrm{S})$ :
}

Nakaye, Yasukazu; Kawai, Jun

\section{CITATION:}

Nakaye, Yasukazu ...[et al]. Si PIN X-ray photon counter. X-Ray

Spectrometry 2011, 40(4): 257-259

\section{ISSUE DATE:}

2011-03-24

URL:

http://hdl.handle.net/2433/197743

\section{RIGHT:}

This is the peer reviewed version of the following article: Nakaye, Y. and Kawai, J. (2011), Si PIN X-ray photon counter. XRay Spectrom., 40: 257-259, which has been published in final form at http://dx.doi.org/10.1002/xrs.1326; この論文は出 版社版でありません。引用の際には出版社版をご確認ご利用ください。; This is not the published version. Please cite only the published version. 


\title{
Si PIN X-ray Photon Counter
}

Yasukazu Nakaye and Jun Kawai

Department of Materials Science and Engineering, Kyoto University, Sakyo-ku, Kyoto 606-8501, Japan.

\begin{abstract}
A Si PIN detector for visible light detection, instead of a Geiger-Müller tube, is applied to X-ray photon counting. We counted radiation from a checking source of a Geiger-Müller counter with a Si PIN counter and with a Geiger-Müller counter. White X-ray of energy up to $20 \mathrm{keV}$ emitted from a pyroelectric X-ray emitter was also counted, and the Si PIN X-ray counter showed a similar curve of count rate versus source distance in both measurements. Pulse counting was performed by spectroscopy circuits. An audio digitizer with computer software for signal processing was also employed to simplify the photon counter. A plot of count rate versus time was obtained with this setup. With simple pulse counting circuits, Si PIN X-ray counters have advantages such as compact structure, low cost and easy application.
\end{abstract}

\section{Introduction}

Today Geiger-Müller counters are most commonly used for radiation monitoring in many scientific and industrial areas. In this paper, we propose a simple detector for counting X-ray photons using a large area Si PIN photodiode (active area is 10 x $10 \mathrm{~mm}$ ) (Fig. 1a, b) instead of a Geiger-Müller tube. Commercially available Si PIN diode chips are prepared by starting with a wafer of almost intrinsically pure silicon, followed by chemical deposition process. Thin p-layer and n-layer is then diffused into each side of diode surface. The resulting middle region is an intrinsic layer. This simple fabrication process makes it easy for us to provide Si PIN. We conducted the following experiments to reveal the current status of Si PIN X-ray counters.

\section{Experimental}


The Si PIN photodiode used in the present work has no coating material on the exposed surface in order to extend the intrinsic response to short wavelength lights. Therefore, it will be easily saturated when it is exposed to ambient visible light. And it takes time to recover to the normal state. Then, we mounted this Si PIN detector on a brass cylinder to prevent it from electrical noise and vibration (Fig. 1a), and we covered the detector with aluminum foil to shield it from visible light (Fig. 1c). The major specifications of this Si PIN detector are as follows: No coating material, active area $10 \times 10$ mm, p-layer thickness $1 \mu \mathrm{m}$, i-, n-layer thickness $300 \mu \mathrm{m}$. To achieve the simplest counting system, we used this Si PIN detector without reverse voltage or thermal cooling.

A simplified circuit diagram of the setup for testing the Si PIN X-ray photon counter with spectroscopy circuits is shown in Fig. 2a. Although it is very simple, it has all components for photon counting. The measurement process is as follows. A Si PIN detector (HAMAMATSU, S3590-09) (Fig. 1a) detects an X-ray photon. The output current pulse elicited from the Si PIN photodiode was applied to a charge sensitive preamplifier (ORTEC, 142B). Then the preamplifier output was transmitted to a linear amplifier (ORETEC, 716A) for shaping, i.e., derivation and integration, then to the Timing Single channel analyzer (SCA) (ORTEC, 551) to determine the presence of a certain pulse height of detected radiation. An SCA is used as a discriminator, so it produces an output logic pulse if its input signal exceeds a preset threshold level. The logic pulse output of the SCA was applied to the Log/Lin ratemeter (ORTEC, 449-2).

To make the X-ray photon counter much simpler, we used signal processor software we developed with audio digitizer (Fig. 2b). As mentioned in the previous work [1], we can replace most of the spectroscopic circuits with our software and audio digitizer on a notebook computer. And also the experimental setup for measurement is reduced its size and numbers. With this setup, we measured $\mathrm{X}$-ray of energy up to $20 \mathrm{keV}$ emitted from a pyroelectric X-ray emitter. The pyroelectric crystal in a pyroelectric X-ray emitter is repeatedly heated and cooled to provide high voltage. As a result of this 
cycle, the emitted X-ray count rate changes periodically. Therefore a pyroelectric X-ray emitter is suitable to check the basic character of the X-ray counter.

\section{Results and Discussions}

To determine the basic character of the counter, we measured the output count rate of a Si PIN counter and a Geiger-Müller counter. We changed the distance between a detector and an X-ray source to change the X-ray flux. Figure 3 shows a comparison of count rate versus distance from a detector to a Ra radio isotope X-ray source (checking source of a Geiger-Müller counter, active area is 10 x $25 \mathrm{~mm}$ ) from the detector. The Si PIN X-ray counter shows 4 times larger count rate than the Geiger-Müller counter at $0 \mathrm{~cm}$ distance. This is because the total photon cross section per $0.3 \mathrm{~mm}$ thickness of solid Si (the thickness of the Si PIN detector) is 5 times larger than the total photon cross section per $130 \mathrm{~mm}$ thickness of Ar gas (the length of the Geiger-Müller tube). [2] As the distance from a detector to the X-ray source increases, the intensity ratio obtained with the Si PIN counter and the Geiger-Müller counter decreases. In other words, as the distance increases, the area illuminated by X-ray source differs from overlapped active area of both detector and X-ray source. From the result that the Si PIN counter and the Geiger-Müller counter showed similar curves of count rate versus source distance, we can say that, with equivalent circuit to Fig. 2a, very simple X-ray counters are achievable.

To clarify both the sensitivity of the Si PIN counter in energy range of up to $20 \mathrm{keV}$ and its stability after repeating exposure to X-ray of the Si PIN counter, we applied the cyclic X-ray emission to the Si PIN counter by means of the pyroelectric X-ray emitter (a spectrum is shown in Fig. 4B). It was shown that the sensitivity for X-ray emission was stable after repeating exposure to X-ray. A plot of count rate versus time obtained by an audio digitizer and software is given in Fig. 4. The repeating cycle of heating phase and cooling phase of a pyroelectric X-ray emitter was obtained. The gradually decreasing intensity of emitted X-ray is due to the continuous decrease of the change in temperature 
per cycle; caused by joule heating of the package of pyroelectric X-ray emitter.

\section{Conclusions}

It has been shown that a Si PIN photodiode for visible light detection is capable of counting X-ray photons without reverse voltage or thermal cooling. These characters allow us to apply Si PIN detectors as a simple X-ray counter instead of a conventional X-ray counter; Geiger-Müller counters. As shown in Fig. 3 we counted X-rays from a Ra radio isotope (active area is 10 x $25 \mathrm{~mm}$ ) with the Si PIN photodiode (10 x $10 \times 0.3 \mathrm{~mm})$ and the Geiger-Müller tube (20 mm in diameter and $130 \mathrm{~mm}$ in length). The experimental result clearly expressed the counting efficiency of the Si PIN photodiode was 4 times larger than that of the Geiger-Müller tube because the photon cross section of $0.3 \mathrm{~mm}$ thick silicon is 5 times larger than that of $130 \mathrm{~mm}$ thick argon gas (Geiger-Müller tube). And from the result of counting X-ray from a pyroelectric X-ray emitter, the capability of the Si PIN X-ray counter of counting low energy X-rays (up to $20 \mathrm{keV}$ ) was shown. The stability of the Si PIN X-ray counter after repeatedly exposed under the X-ray was also certified. 


\section{References}

[1] Y. Nakaye, J. Kawai, X-ray Spectrum., 39, pp.318-320, 2010.

[2] M. J. Berger, J.H. Hubbell, S. M. Seltzer, J. Chang, J. S. Coursey, R. Sukumar and D. S. Zucker, XCOM: Photon Cross Section Database (Version 3.1). Gaithersburg, MD: National Institute of Standards and Technology, 2009. 
Figure captions

Fig. 1: Photographs of the Si PIN semiconductor detector (HAMAMATSU, S3590-09) used in the present experiment. a) is the detector chip, and b) shows the chip fixed on the brass cylinder and on the BNC connector. c) is a photograph of the detector connected to the preamplifier (ORTEC, 142B) and covered with aluminum foil to shield it from the visible light.

Fig. 2: a) A simplified circuit diagram of a Si PIN X-ray counter with spectroscopy circuits and b) the Si PIN X-ray counter with audio digitizer. Each number in triangle symbols represents the ORTEC model number.

Fig. 3: Comparison of the count rate per square millimeter of X-ray counter with a Si PIN (filled circle and solid line) and a Geiger-Müller tube (open circle and dashed line). The count rate was divided by overlapped area of Ra radio isotope and each detector's active area at $0 \mathrm{~cm}$ distance.

Fig.4: A) A plot of count rate versus time obtained with the Si PIN X-ray counter. The repeating cycle of heat phase and cool phase of a pyroelectric X-ray emitter was obtained. X-ray flux from the X-ray emitter decreased as thermal cycles repeated. B) A spectrum of X-ray emitted from the pyroelectric X-ray emitter (30 heating-cooling cycles). 

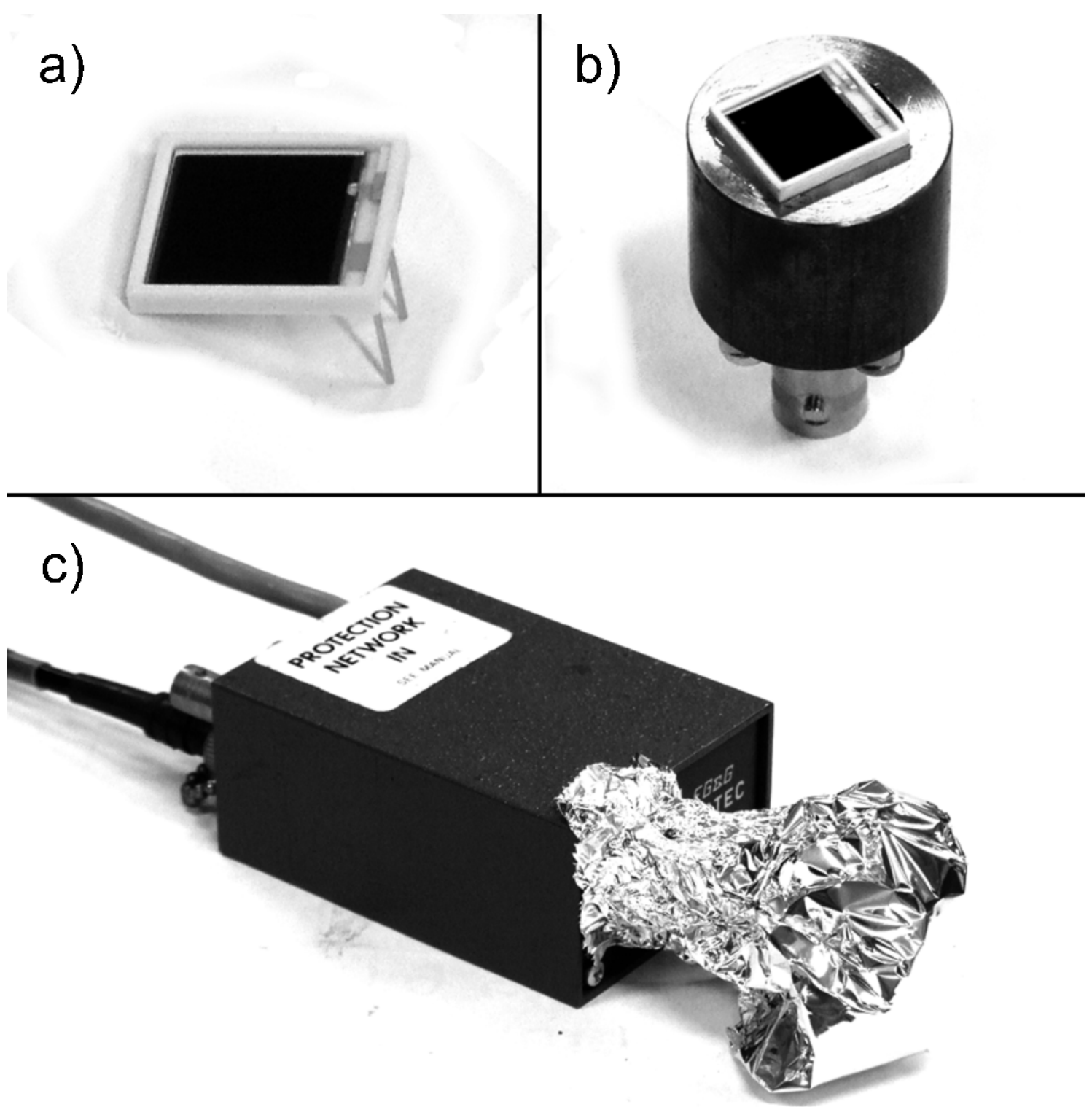

Fig. 1 
a)

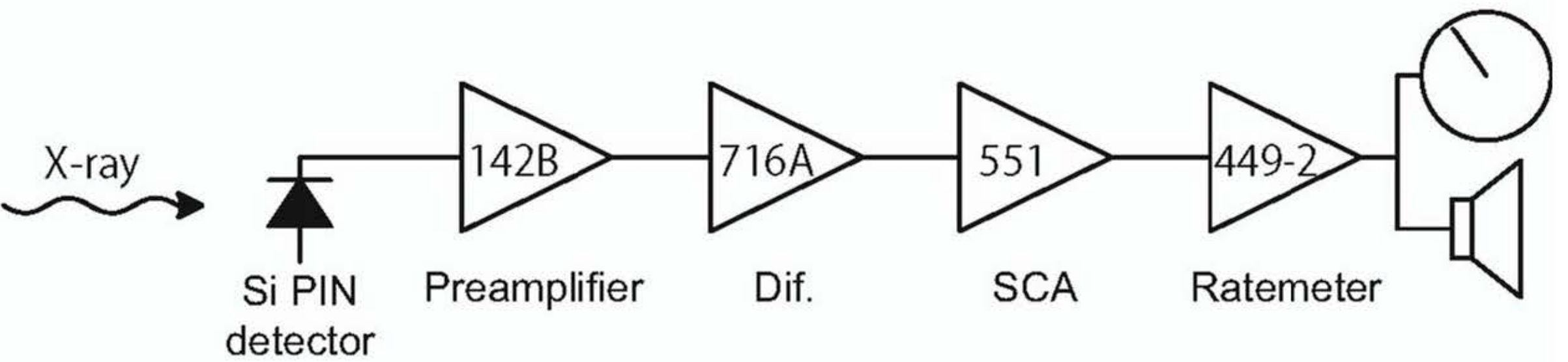

b)

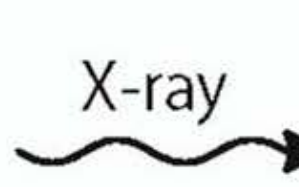

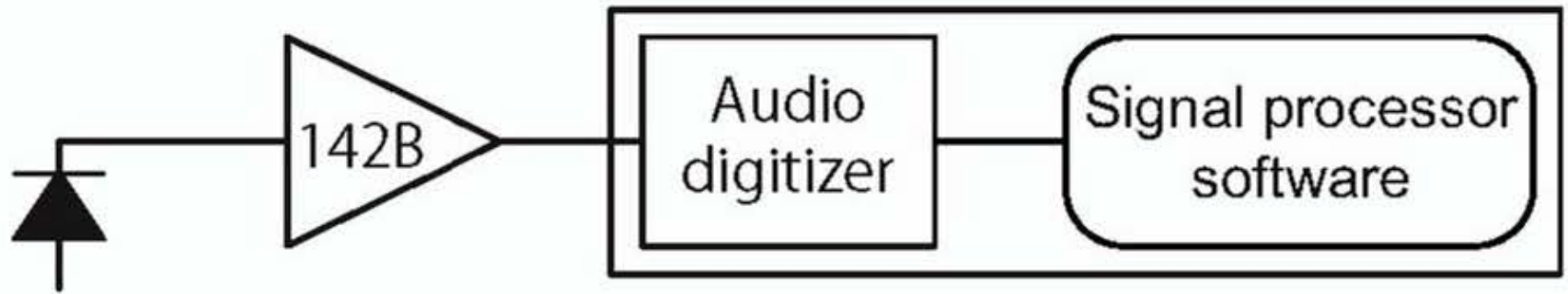

Notebook Computer
Fig. 2 


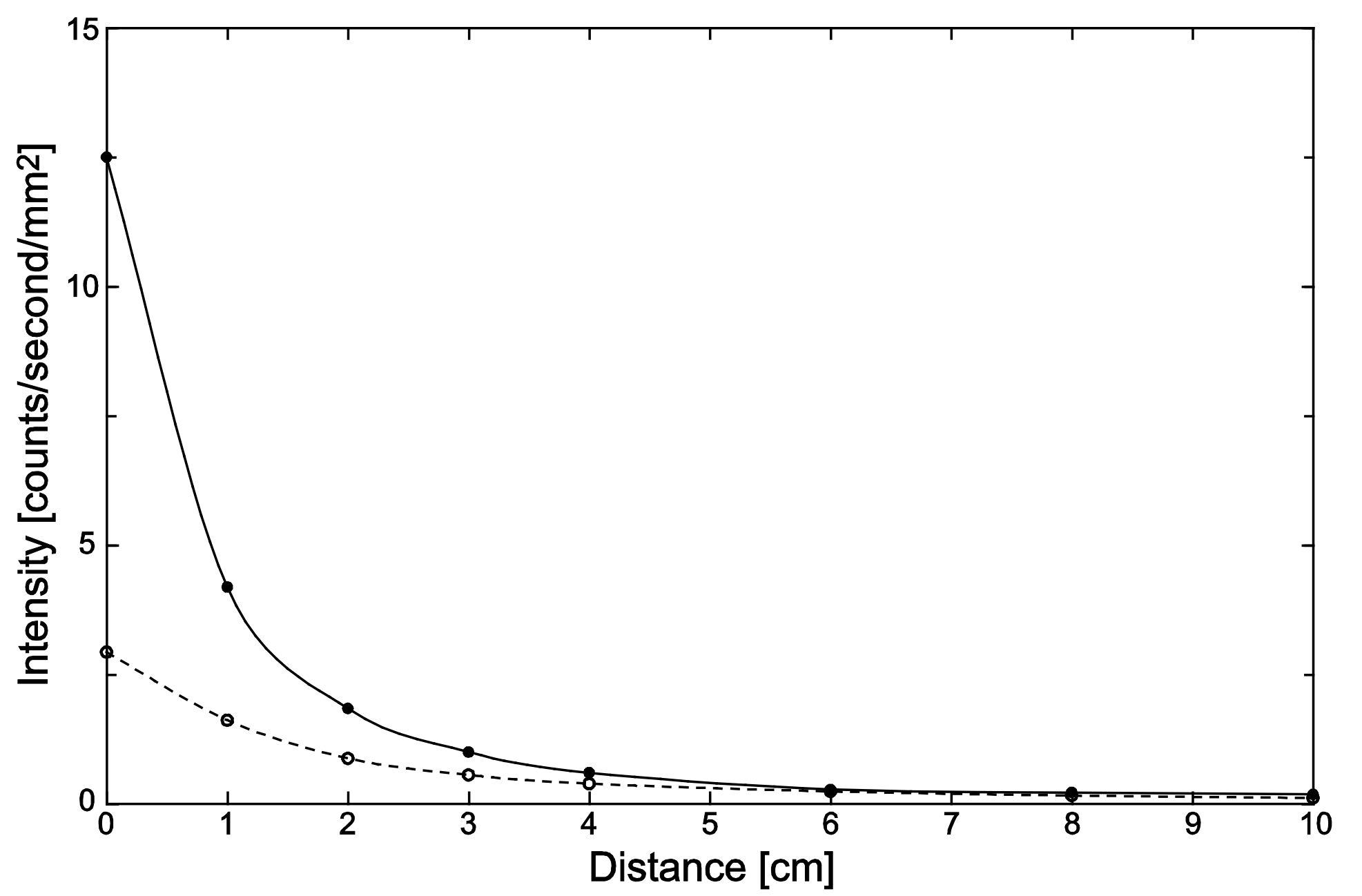

Fig. 3 


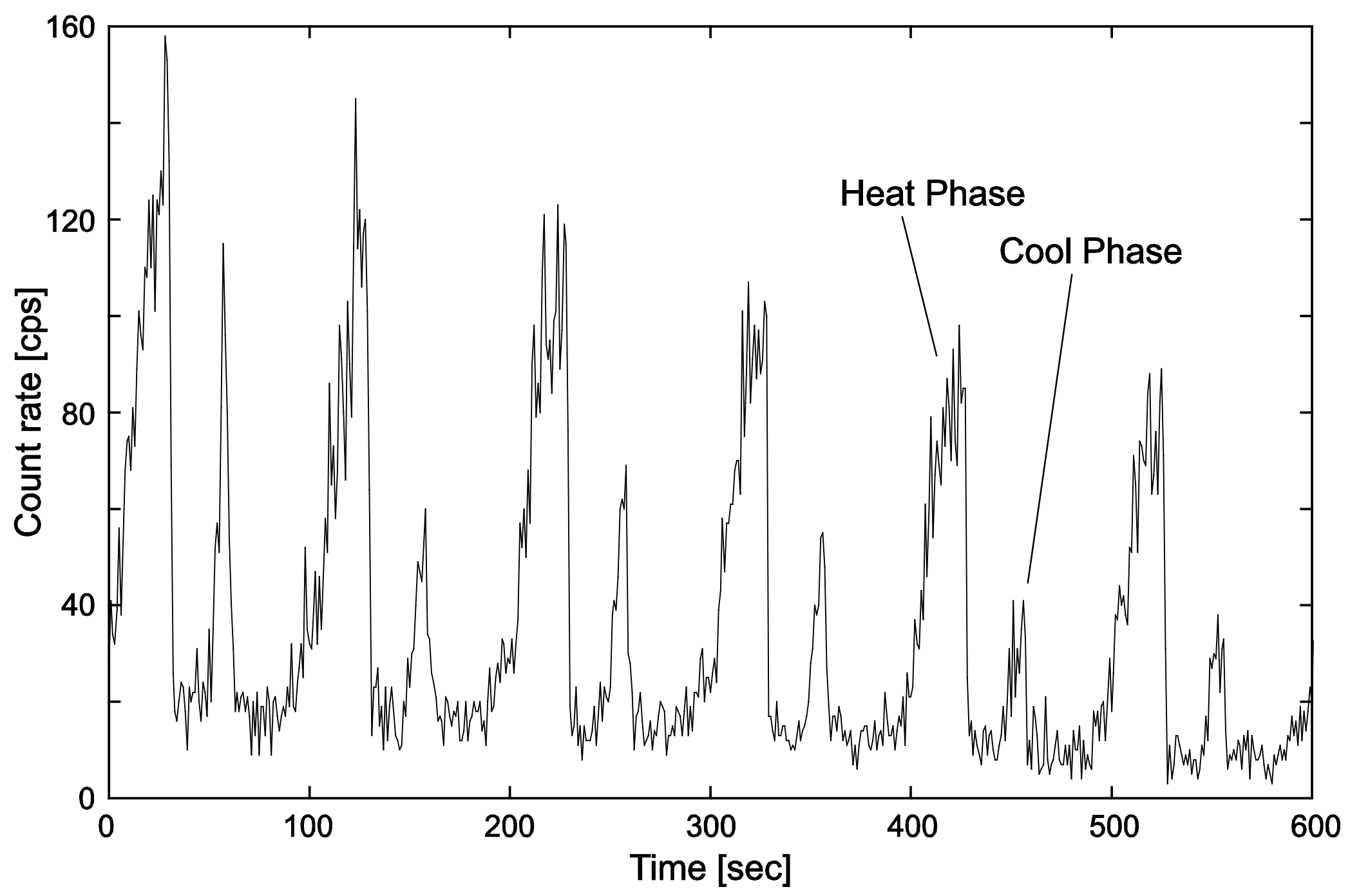

Fig. 4 